\title{
Controllability with Unilateral Control Inputs
}

\author{
Bill Goodwine and Joel Burdick \\ Division of Engineering and Applied Science \\ California Institute of Technology \\ Mail Code 104-44, Pasadena, CA 91125 \\ Email: goodwine@robby.caltech.edu, jwb@robby.caltech.edu
}

\begin{abstract}
Many control systems contain control inputs which are constrained to be non negative. Unfortumately, the unilateral nature of such inputs makes them similar to a drift term, and correspondingly complicates any attempt to determine controllability. We present a controllability test in which Lie brackets are characterized as "good" or "bad," from which controllability follows from the relationship between these "good" and "bad" brackets. This test is relatively straightforward to apply and its application is illustrated with an example.
\end{abstract}

\section{Introduction}

Many interesting and useful control systems have control inputs which are constrained to be nonnegative; yet, the issue of controllability of such systems has not been fully explored. For example, a control input corresponding to some sort of thruster can only produce a zero or positive thrust (unless the thrusters are arranged in opposing pairs). Similarly, a control input corresponding to the normal force produced by physical contact clearly must also be non negative. Such milateral forces arising from physical contact naturally arise in many locomotion problems, wherein an object, such as an animal or robot, moves through its environment via physical contact with surrotunding objects.

To our knowledge, the few fundamental results pertaining to controllability for such restricted control inputs appear in work by Sussmann in $[1,2]$ and by Lynch and Mason in [3]. The results by Sussmann in references $[1,2]$ related to the case where the inputs are restricted to be non negative, are low order tests, i.e., the Lie algebra rank condition must be satisfied by vector fields of sufficiently low order. We present a result which is distinct to the extent that it allows the Lie algebra rank condition to be satisfied by vector fields of arbitrarily high order. However, this comes at the expense of a more restrictive condition on the first order vector fields.

\section{Unilateral Control Inputs}

We will consider control systems of the form

$$
\dot{x}=f(x)+h_{i}(x) v^{i}+g_{j}(x) u^{j}
$$

where $i=1, \ldots, m, j=1, \ldots, n, v^{i} \in[0,1) \forall i, u^{j} \in$ $(-1,1) \forall j, x \in M, M$ a manifold and $f, h_{i}$ and $g_{j}$ 0-7803-3590-2/96 \$5.00 다 1996 IEEE vector fields on $M$; that is, the control inputs $v^{i}$ are restricted to be non negative.

Let $\mathcal{U}$ be the set of admissible controls. Given an open set $V \subseteq M$, define $R^{V}\left(x_{0}, T\right)$ to be the set of states $x$ such that there exists $\left(v^{i}, u^{j}\right):[0, T] \rightarrow \mathcal{U}$ that steers the control system from $x(0)=x_{0}$ to $x(T)=x_{f}$ and satisfies $x(t) \in V$ for $0 \leq t \leq T$. Define

$$
R^{V}\left(x_{0}, \leq T\right)=\bigcup_{0<\tau \leq T} R^{V}\left(x_{0}, \tau\right)
$$

to be the set of states reachable up to time $T$. A system is small time locally controllable ("STLC") if $R^{V}\left(x_{0}, \leq\right.$ $T)$ contains a neighborhood of $x_{0}$ for all neighborhoods $V$ of $x_{0}$ and $T>0$.

Let $\mathcal{C}$ be the smallest subalgebra of $V^{\infty}(M)$ (the Lie algebra of smooth vector fields on $M$ ) that contains $f, h_{1}, \ldots, h_{m_{i}}, g_{1}, \ldots, g_{n}$, and let $C$ be the accessibility distribution generated by $\mathcal{C}$ :

$$
C(x)=\operatorname{span}\{X(x): X \in \mathcal{C}\}, \quad x \in M
$$

If $\operatorname{dim} C\left(x_{0}\right)=\operatorname{dim} M$, then the system satisfies the Lie Algebra Rank Condition ("LARC") at $x_{0}$.

For a given Lie bracket $X$, consider the degree of a bracket with respect to a vector field $f, h_{i}$ or $g_{j}$, denoted by $\delta^{f}(X), \delta^{h_{i}}(X)$ and $\delta^{g_{j}}(X)$, respectively, to be the number of times that the superscripted vector field appears in the bracket $X$. Now consider the total degree, $\delta(X)$, to be

$$
\delta(X)=\delta^{f}(X)+(1+\epsilon) \sum_{i=1}^{n_{2}} \delta^{h_{i}}(X)+\sum_{j=1}^{n_{2}} \delta^{g_{j}}(X)
$$

where $0<\epsilon \ll 1$. This concept of degree and total degree is presented rigorously in Section 4, but for now we take it to mean that we can determine the degree of a bracket by comnting the number of times that a particular vector field appears in the bracket.

Now, we will call a bracket, $X$ "bad" if $\delta^{s j}$ is even (including 0 ) for each $j, \delta^{f}(X)+\sum_{i=1}^{T b} \delta^{h_{i}}(X)$ is odd and $\sum_{i=1}^{m} \delta^{h_{i}}(X) \neq 1$. Otherwise, call the bracket "good."

We can now state our main result. 
Proposition 2.1 Consider the control system described by Equation 1. Assume that the system satisfies the LARC and that there exist coefficients $\lambda_{i}$ and $\alpha_{j}$ such that

$$
\sum_{i=1}^{m} \lambda_{i} h_{i}(x)+\sum_{j=1}^{m} \alpha_{j} g_{j}(x)=0 \quad \forall x \in \operatorname{nbd}\left(x_{0}\right),
$$

where $\lambda_{i} \in(0,1)$ and $\alpha_{j} \in \mathbb{R}$. Assume further that any bad bracket can be written as a linear combination of brackiets of lower total degree. Then the system is $S T L C$ at $x_{0}$.

The proof of this Proposition appears in Section 5. We note that in the proof of this result, we provide an alternative result given by Proposition 5.1 which is more general and thus less restrictive; however, in practice, this more general test will be more difficult to apply than Proposition 2.1, above.

Although technically difficult to prove, the intuition behind the restriction expressed by Equation 2 is simple. Due to the control input restrictions, none of the control inputs $v^{i}$ can be negative. However, Equation 2 can be solved for one $-h_{i}$ in terms of the other $h_{j}$ 's with positive coefficients and the $g_{k}$ 's with arbitrary coefficients. Thus, allowable control inputs $\left(v^{j}>0, j \neq i\right)$ effect the same result as a disallowed control input (one $\left.v^{i}<0\right)$.

\section{Example}

Before we present the proof of Proposition 2.1, we will illustrate its application with an example. Here we consider the controllability of rigid body with "thrusters." Initially consider the body to be centered about orthogonal coordinate axes, and let two thrusters be placed at the points where the $x$-axis intersects the surface of the body and two more thrusters placed at the points where the $y$-axis intersects the surface. Let the force of the thrusters on the $x$ axis be in the negative $z$ direction, and the force of the thrusters on the $y$ axis be in the positive $z$ direction. As a fifth control input, let the thrusters rotate by a small angle, $\psi$ (which can be both positive and negative) about their respective axes so that the thrusters aligned on the $x$ axis rotate in opposite directions so that if they are both "thrusting" they both contribute to a positive torque about the $z$ axis, and let the thrusters aligned on the $y$-axis rotate in opposite directions so that both contribute a negative torque about the $z$ axis. We will consider a spherical body with unit radius and mass $\frac{5}{2}$ (so that the inertia tensor is the identity) as illustrated in Figure 1; although, for a non spherical body, the following controllability analysis still holds. In Figure 1 , for clarity, the coordinate system is shown displaced from the center of mass of the sphere, but for the calculations, the origin of the coordinate system is assumed to initially coincide with its center of mass.

Parameterize the configuration space for the system $S E(3) \times S^{1}$ by the coordinates $X=(x, y, z)$, which

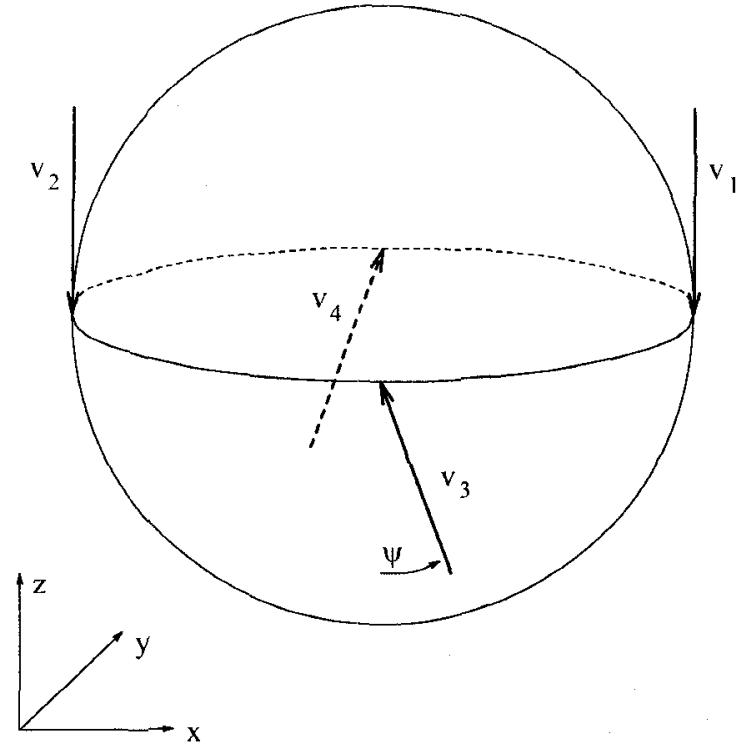

Figure 1. Rigid Body with Thrusters

are the displacements of the center of mass from the fixed inertial frame, $\Phi=\left(\phi_{1}, \phi_{2}, \phi_{3}\right)$, which are the "roll, pitch, yaw" rotations about the $x-y$ and $z$ axes, respectively and $\psi$, which is the rotational angle of the thrusters. Thus, a point in the phase space is given by $q=(X, \Phi, \dot{X}, \dot{\Phi}, \psi)$.

Now, we can write the equations of motion as

$$
\left(\begin{array}{c}
\dot{X} \\
\dot{\Phi} \\
\ddot{X} \\
\ddot{\Phi} \\
\dot{\psi}
\end{array}\right)=\left(\begin{array}{c}
\dot{X} \\
\dot{\Phi} \\
0 \\
-Q^{-1} \dot{Q} \dot{\Phi} \\
0
\end{array}\right)+F \cdot v
$$

where $Q$ is the local mapping which takes the derivatives of the roll, pitch and yaw coordinates we chose. for our parameterization and gives the body angular velocities, which given by

$$
Q=\left(\begin{array}{ccc}
\cos \phi_{2} \cos \phi_{3} & \sin \phi_{3} & 0 \\
-\cos \phi_{2} \sin \phi_{3} & \cos \phi_{3} & 0 \\
\sin \phi_{2} & 0 & 1
\end{array}\right)
$$

If we let $\hat{e}_{1}, \hat{e}_{2}$ and $\hat{e}_{3}$ represent the standard unit vectors in the $x, y$ and $z$ directions fixed in the body, $R_{x}$ and $R_{y}$ represent the usual rotation matrices representing a rotation by an angle $\psi$ about the $x$ axis and the $y$ axis, respectively and $R$ represent the $3 \times 3$ rotation matrix that takes body coordinates to spatial 
coordinates, then

$$
\begin{gathered}
F=\left(\begin{array}{ccc}
0 & 0 \\
0 & 0 & \\
-\frac{5}{2} R R_{x} \hat{e}_{3} & -\frac{5}{2} R R_{x} \hat{e}_{3} & \\
Q^{-1}\left(\left(R_{x} \hat{e}_{3}\right) \times \hat{e}_{2}\right) & Q^{-1}\left(\left(-R_{x} \hat{e}_{3}\right) \times \hat{e}_{2}\right) \\
0 & 0 & \\
0 & 0 & 0 \\
0 & 0 & 0 \\
\frac{5}{2} R R_{y} \hat{e}_{3} & \frac{5}{2} R R_{y} \hat{e}_{3} & 0 \\
Q^{-1}\left(\left(R_{y} \hat{e}_{3}\right) \times e_{1}\right) & \left(Q^{-1}\left(-R_{y} \hat{e}_{3}\right) \times e_{1}\right) & 0 \\
0 & 0 & 1
\end{array}\right)
\end{gathered}
$$

and $v=\left(v^{1}, \ldots, v^{4}, u\right)^{T}$. We will refer to the first four columms in the matrix on the right hand side of the equation for $F$ as $h_{1}, \ldots, h_{4}$, the fifth colimn as $g$ and the first column on the right hand side of Equation 3 as $f$, to notationally correspond to Equation 1, i.e., the thruster forces, $v_{j}$ are restricted to be non-negative, and the thruster rotation angle, $\psi$ can be either positive or negative.

First we must check that the system satisfies the LARC. Tedions calculations show that the following collection of vector fields spans $T_{x} M$ everywhere except for the parameterization singularity at $\phi_{2}=\frac{\pi}{2}$ :

$$
\begin{gathered}
\left\{h_{1}, h_{2}, h_{3}, g,\left[g, h_{1}\right],\left[g, h_{2}\right],\left[h_{1}, f\right],\left[h_{2}, f\right],\left[h_{3}, f\right],\right. \\
\left.\left[g, h_{3}\right],\left[\left[g, h_{1}\right], f\right],\left[\left[g, h_{2}\right], f\right],\left[\left[g, h_{3}\right], f\right]\right\} .
\end{gathered}
$$

Now, we note that the hypothesis of Proposition 2.1 expressed by Equation 2 is satisfied because

$$
\sum_{j} h_{j}(x)=0, \quad \forall x \in M .
$$

The LARC is satisfied by brackets with total degree $\leq 3+\epsilon$, so we need to show that all bad brackets with total degree $\leq 3+\epsilon$ are spanned by brackets with lower total degree. Note, that the only bad bracket, with one element is $f(x)$. However, $f(x)=0$ if $\dot{X}=\dot{\Phi}=0$. For brackets with three elements, we note that any bad bracket must have zero or two occurrences of the vector field $g$. If there are zero $g$ 's, there mist be one or more $h$ 's (since $[f,[f, f]]=0$ ). If there is only one $h$, then it is not a bad bracket. If there are two or more $h$ 's, then the total degree of the bracket is greater than $3+\epsilon$. If there are two occurrence of $g$, then there must either be two $g$ 's and one $h_{i}$ or two $g$ 's and one $f$. In the first case, since there is one $h_{i}$, that bracket is not a bad bracket. In the second case, we note that in this example $[g, f](x)=0$, so that bad bracket can be written as a linear combination of lower order elements. Therefore the system is STLC from any position with zero velocity.

\subsection{Simulation Results}

In this section we present sinnulation results which demonstrate the controllability properties of the rigid body example. The following graphs are intended to illustrate that, after a sequence of control imputs, and possibly after a complicated series of gyrations, the system, to leading order, has undergone a net motion in a particular direction. We only present results for motion in two directions. but note that it is possible to do so for all directions in the 13 dimensional phase space.

For example, consider motion in the $x$-direction. We note that $\dot{x}=\frac{1}{2}\left[\left[g . h_{1}\right], f\right]-\frac{1}{2}\left[\left[g . h_{2}\right], f\right]$. Figure 2 illustrates a sequence of motions that the system may undergo to move in the $x$ direction. Note that the system is momentarily displaced in varions states other than purely the $x$-direction: however. the end result is pure displacement in the $x$ direction.

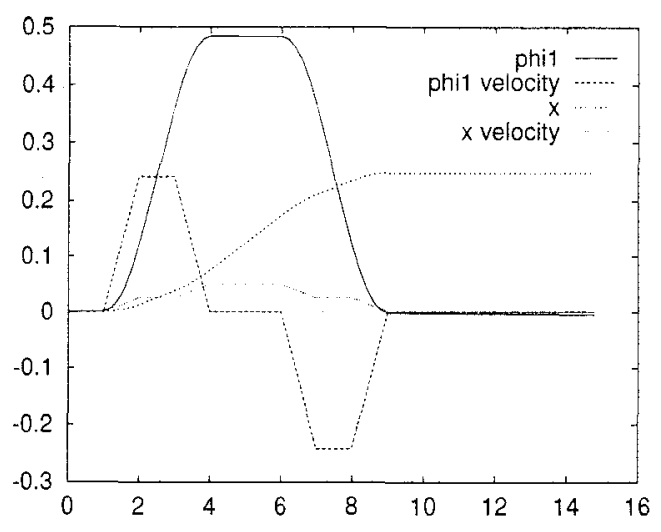

Figure 2. Control Inputs for $x$ Direction

Also, consider motion in the $\phi_{3}$ direction. Note that $\dot{\phi}_{3}=\frac{1}{5}\left(\left[\left[g, h_{1}\right], f\right]+\left[\left[g, h_{2}\right], f\right]\right)$. Figure 3 illustrates a sequence of motions that the system may undergo to move in the $\phi_{3}$-direction. Again, note that the system is momentarily displaced in various states other than purely the $\phi_{3}$ direction: however, the end result is pure displacement in the $\phi_{3}$ direction.

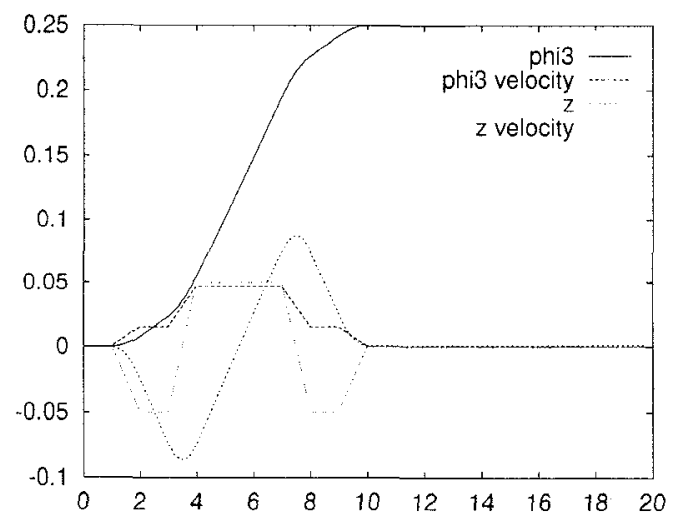

Figure 3. Control Inputs for $\phi_{3}$ Direction

Rather than specifically illustrate how we obtained the sequence of control inputs used for the above simulations, we will discuss the hetristic synthesis technique we used in more general terms. This technique is merely presented to illustrate the means by which we obtained the control inputs for the above examples, and is not presented as part of a rigorous theory. (A possible rigorous approach to this synthesis problem would be to extend the averaging results of Leonard 
and Krishnaprasad [?] to the context of the problem we are considering.)

Recall that if we denote the flow of a vector field $f$ at time $t$ starting at a point $x_{0}$ by $\phi_{t}^{f}\left(x_{0}\right)$, we can write

$$
\phi_{\epsilon}^{-g_{2}} \cdot \phi_{\epsilon}^{-g_{1}} \cdot \phi_{\epsilon}^{g_{2}} \cdot \phi_{\epsilon}^{g_{1}}\left(x_{0}\right)=x_{0}+\epsilon^{2}\left[g_{1}, g_{2}\right]+\mathcal{O}\left(\epsilon^{3}\right),
$$

where $\phi_{\epsilon}^{g} \cdot \phi_{\epsilon}^{f}$ denotes the composition of the two flows, i.e., the flow along an integral curve of $f$ followed by the flow along an integral curve of $g$. Thus to "flow" in the direction of a Lie bracket (to leading order), we simply modulate the control inputs associated with two vector fields to execute the sequence of flows illustrated.

Now consider, for example, the third order bracket $[f,[f, g]]$. Writing this in terms of its approximation by flows we have

$$
\begin{aligned}
& {[f,[f, g]] \sim} \\
& \quad \phi_{\epsilon}^{-f} \cdot \phi_{\epsilon}^{-g} \cdot \phi_{\epsilon}^{f} \cdot \phi_{\epsilon}^{g} \cdot \phi_{\epsilon}^{-f} \cdot \phi_{\epsilon}^{-g} \cdot \phi_{\epsilon}^{-f} \cdot \phi_{\epsilon}^{g} \cdot \phi_{\epsilon}^{f} \cdot \phi_{\epsilon}^{f} .
\end{aligned}
$$

All the $-f$ terms appearing throughout the above equation are clearly problematic. However, it is actually possible to rewrite the bracket in a manner that will allow it to be executed. First, consider

$$
\begin{aligned}
& {[f,[f, g]]=[-f,[-f, g]] \sim} \\
& \phi_{\epsilon}^{f} \cdot \phi_{\epsilon}^{-g} \cdot \phi_{\epsilon}^{-f} \cdot \phi_{\epsilon}^{g} \cdot \phi_{\epsilon}^{f} \cdot \phi_{\epsilon}^{-g} \cdot \phi_{\epsilon}^{f} \cdot \phi_{\epsilon}^{g} \cdot \phi_{\epsilon}^{-f} \cdot \phi_{\epsilon}^{-f} .
\end{aligned}
$$

Now, the first two $-f$ terms do not affect the flow since we assume that $f\left(x_{0}\right)=0$. There still is one remaining $-f$ flow. However, we note that it corresponds to a set of four flows which are approximating the bracket $[g,-f]$. However, $[g,-f]=[-f,-g]$ and $[-f, g] \sim$ $\phi_{\epsilon}^{y \prime} \cdot \phi_{\epsilon}^{f} \cdot \phi_{\epsilon}^{-g} \cdot \phi_{\epsilon}^{-f}$, and when this is substituted into Equation 4, we have

$$
\begin{aligned}
& {[f,[f, g]]=[-f,[-f, g]] \sim} \\
& \quad \phi_{\epsilon}^{g} \cdot \phi_{\epsilon}^{f} \cdot \phi_{\epsilon}^{-g} \cdot \phi_{\epsilon}^{-f} \cdot \phi_{\epsilon}^{f} \cdot \phi_{\epsilon}^{-g} \cdot \phi_{\epsilon}^{f} \cdot \phi_{\epsilon}^{g} \cdot \phi_{\epsilon}^{-f} \cdot \phi_{\epsilon}^{-f},
\end{aligned}
$$

and so the composed flow $\phi_{\epsilon}^{-f} \cdot \phi_{\epsilon}^{f}=0$, and thus has no effect on the net flow. Finally, to flow along the negative direction of an integral curve of one of the $h_{i}$, we simply flow along the positive direction of the vector field $\sum_{j \neq i} h_{j}$.

These observations allowed us to determine sequences of control inputs which produced displacements in all 13 states of the system, similar to the results illustrated in Figures 2 and 3

\section{Background Material}

In this Section, we briefly outline the necessary mathematical objects for the proof. In Section 5, we present the proof for the main result. This presentation is necessarily brief. Complete definitions and more complete explanations of these can be found in Sussmann [5] or Lewis [6]. Other algebraic material is from $[7,8]$.

Denote the free associative algebra over $\mathbb{R}$ generated by the set of non commuting incleterminates $\mathbf{X}=\left\{X_{0}, X_{1}, \ldots, X_{m+n}\right\}$ by $A(\mathbf{X})$ and its homogeneous components of degree $N$ by $A^{N, h_{0 m}}(\mathbf{X})$. Let $L(\mathbf{X})$ be the free Lie algebra generated by $\mathbf{X}$, and denote its homogeneous components of degree $N$ by $L^{N, h o m}(\mathbf{X})$.

Let $\hat{A}(\mathbf{X})$ denote the set of all formal power series $\sum_{I} a_{I} X_{I}$, where $X_{I}=X_{i_{1}} X_{i_{2}} \cdots X_{i_{k}}$ for the multi index $I=\left(i_{1}, i_{2}, \ldots, i_{k}\right)$ and let $\hat{A}_{0}(\mathrm{X})$ denote the set of formal power series for which $a_{\varphi}=0$. The exponential map is the well defined bijection

$$
\exp : \hat{A}_{0}(\mathbf{X}) \rightarrow 1+\hat{A}_{0}(\mathbf{X})
$$

whose inverse is denoted by $\log$, both of which are defined by their usual series definitions.

Also, let $\hat{L}(\mathbf{X}) \subseteq \hat{A}_{0}(\mathbf{X})$ be the set of all formal sums $\sum_{N=1}^{\infty} S_{N}$ such that each $S_{N}$ is in $L^{N, h o m}(\mathbf{X})$. Note that the exponential map is well defined on $\hat{L}(\mathbf{X})$. The elements of $\hat{A}(\mathbf{X})$ that are of the form $\exp (S)$ for some $S \in \hat{L}(\mathbf{X})$ are the exponential Lie series in $X_{0}, \ldots, X_{m}$. The set of all such series is denoted by $\hat{G}(\mathbf{X})$, which is a group. The exponential map, restricted to $\hat{L}(\mathbf{X})$ is a bijection from $\hat{L}(\mathbf{X})$ to $\hat{G}(\mathbf{X})$.

Consider the differential equation

$$
\dot{S}=S\left(X_{0}+\sum_{i=1}^{m} u_{i} X_{i}\right)
$$

for an $\hat{A}(\mathbf{X})$-valued function $t \rightarrow S(t)$, with the initial condition $S(0)=1$. Sussmann [5] notes that the solution to this differential equation exists and is unique, and is given by

$$
S(t)=\sum_{I}\left(\int_{0}^{t} u_{I}\right) X_{I}
$$

where

$$
\begin{aligned}
& \int_{0}^{t} u_{I}=\int_{0}^{t} \int_{0}^{\tau_{k}} \int_{0}^{\tau_{k-1}} \cdots \\
& \quad \int_{0}^{\tau_{2}} u_{i_{k}}\left(\tau_{k}\right) u_{i_{k-1}}\left(\tau_{k-1}\right) \cdots u_{i_{1}}\left(\tau_{1}\right) d \tau_{1} \cdots d \tau_{k} .
\end{aligned}
$$

The series $S(T(u(\cdot)))$, with $t \rightarrow S(t)$ as above is the formal power series associated with the control $u(\cdot)$, and will be denoted by $\operatorname{Ser}(u(\cdot))$, where $T(u(\cdot))$ is the terminal time of $u(\cdot)$.

Let $\mathcal{U}$ be the set of all functions $u(\cdot)$ whose domain is a compact interval of the form $[0, T]$ such that $u(\cdot)$ takes values in $\mathbb{R}^{m}$ and is Lebesgue integrable on $[0, T]$. Now, if $K$ is an arbitrary subset of $\mathbb{R}^{m b}$, then we can consider $\mathcal{U}_{m}(K)$, the subsemigroup of $\mathcal{U}_{m}$ whose elements are the $K$ valued controls. The image of $\mathcal{U}_{m}(K)$ under Ser will be denoted by $\hat{S}(\mathbf{X}, K)$.

If $V$ is a linear space over $\mathbb{R}$, a group of dilations of $V$ is a mapping $\rho \rightarrow \Delta(\rho)$ that assigns to every real $\rho>0$ a linear endomorphism $\Delta(\rho): V \rightarrow V$, in such a way that

1. $\Delta(1)=$ identity,

2. $\Delta\left(\rho_{1}\right) \Delta\left(\rho_{2}\right)=\Delta\left(\rho_{1} \rho_{2}\right) \quad \forall \rho_{1}, \rho_{2}$,

3. $V$ has a direct, sum decomposition $V=\oplus V_{j}$ such that the subspaces $V_{j}$ are invariant under the $\Delta(\rho)$, and the action of $\Delta(\rho)$ on each $V_{j}$ is given by multiplication by $\rho^{\alpha_{j}}$ for some $\alpha_{j} \geq 0$. 
Note that any $v \in V$ can be expressed in a unique way as a $\operatorname{sum} \sum_{j} v_{j}, v_{j} \in V_{j}$. The $\Delta$ degree of $v$ is the largest $\alpha_{j}$ such that $v_{j} \neq 0$. A group of dilations is called strict if it has no component of degree zero. We say that $\Delta$ is compatible with $\hat{S}(\mathbf{X}, K)$ if $\Delta(\rho)\left(X_{0}+\sum_{i=1}^{m} u_{i} X_{i}\right)$ is of the form $T\left(X_{0}+\sum_{i=1}^{m} v_{i} X_{i}\right)$ for some $T>0$, where $\left(v_{1}, \ldots, v_{m}\right) \in K$, whenever $0<\rho \leq 1$ and $\left(u_{1}, \ldots, u_{m}\right) \in K$.

If $\mathbf{f}=\left(f_{0}, \ldots, f_{m}\right)$ is an $(m+1)$ tuple of $C^{\infty}$ vector fields on a $C^{\infty}$ manifold $M$, then define the map $\operatorname{Ev}(\mathbf{f})$ which assigns to every element of $L(\mathrm{X})$ the vector fields obtained by plugging in each $f_{i}$ for the corresponding $X_{i}$. If $p \in M$, then define the $\operatorname{map} \operatorname{Ev}_{p}(\mathbf{f})$ from $L(\mathbf{X})$ to $T_{p} M$, given by $\operatorname{Ev}_{p}(\mathbf{f})(Z)=\operatorname{Ev}(\mathbf{f})(Z)(p)$. If $Z \in L(\mathbf{X})$ is $\Delta$-homogeneous, we say that $Z$ is $\Delta$-neutralized for $\mathbf{f}$ at $p$ if $\operatorname{Ev}_{p}(\mathbf{f})(Z)$ can be expressed as a sum of vectors $\operatorname{Ev}_{p}(\mathbf{f})\left(Q_{i}\right)$, where the $Q_{i}$ are elements of $L(\mathbf{X})$ of lower $\Delta$ degree than $Z$. We will refer to the triple $(M, \mathbf{f}, K)$ as a control system.

The class of controls is embedded as a subsemigroup $\hat{S}(\mathbf{X}, K)$ of the group $\hat{G}(\mathbf{X})=\{\exp (Z): Z \in \hat{L}(\mathbf{X})\}$. An automorphism $\lambda$ of $L(\mathbf{X})$ gives rise to a mapping $\hat{\lambda}$, where, if $Z=\sum_{i=1}^{\infty} P_{i}$, where $P_{i}$ is homogeneous of degree $i$, then $\hat{\lambda}(Z)=\sum_{i=1}^{\infty} \lambda\left(P_{i}\right)$. Also, define $\lambda^{\#}$ from $\hat{G}(\mathbf{X})$ to $\hat{G}(\mathbf{X})$ by letting $\lambda^{\#}(\exp (Z))=\exp (\hat{\lambda}(Z))$ for $Z \in \hat{L}(\mathbf{X})$.

An input symmetry is an automorphism $\lambda$ of $L(\mathbf{X})$ such that the corresponding map $\lambda^{\#} \operatorname{maps} \hat{S}(\mathrm{X}, K)$ to $\hat{S}(\mathbf{X}, K)$. A linear map $\lambda: L(\mathbf{X}) \rightarrow L(\mathbf{X})$ is graded if $\lambda$ maps $L^{j, h o m b}(\mathbf{X})$ into $L^{j, h o r r}(\mathbf{X})$ for each $j$.

Finally, we say that an element of $L(\mathrm{X})$ is totally odd if all its homogeneous components have odd degree.

Our result will follow from the following theorem, which is a corollary to the main result in [2].

THEOREM 4.1 Let $(M, \mathbf{f}, K)$ be a control system, and let $p \in M$. Assume that $\mathbf{f}$ satisfies the $L A R C$ at $p$, and that there exists (a) an admissible group of dilations $\Delta$ of $L^{1, \text { hom }}(\mathbf{X})$ which is compatible with $\hat{S}(\mathbf{X}, K)$, (b) a finite group $\Lambda_{0}$ of graded linear maps from $L(\mathrm{X})$ to $L(\mathbf{X})$ that are input symmetries, such that every totally odd $\Lambda_{0}$ fixed element of $L(\mathbf{X})$ is $\Delta$ neutralized for $\mathbf{f}$ at p. Then $(M, \mathbf{f}, K)$ is $S T L C$ from $p$.

\section{Proof of Proposition 2.1}

We will prove Proposition 2.1 as a corollary to a more general result.

Let, $\mathbf{X}=\left\{X_{0}, X_{1}, \ldots, X_{r n}, X_{r b+1}, \ldots X_{r n+n}\right\}$ and $\mathbf{f}=\left\{f, h_{1}, \ldots, h_{u_{2}}, g_{1}, \ldots, g_{n}\right\}$ so that $\operatorname{Ev}(f)$ takes $X_{0}$ to $f, X_{i}$ to $h_{i}$ for $i=1, \ldots, m$ and $X_{j}$ to $g_{j-m}$ for $j=m+1, \ldots, m+n$. The $f, h_{i}$ and $g_{j}$ above corcespond to the vector fields in the Equation 1. Let $\operatorname{Br}(X)$ be the set of "brackets" of elements from $\mathbf{X}$ and $\delta^{i}(B)$ be the number of occumences of $X_{i}$ in $B \in \operatorname{Br}(\mathbf{X})$.

Consider the antomorphism generated by $\sigma_{i}, i=$ $m+1, \ldots, m+n$ where $\sigma_{i}$ sends $X_{j}$ to $X_{j}$ if $j \neq i$ and
$X_{i}$ to $-X_{i}$. Clearly, a $\Lambda_{0}$ fixed element of $L(\mathbf{X})$ camnot have an odd number of each $X \in\left\{X_{n+1}, \ldots, X_{m+n}\right\}$. Thus, we can consider only elements with an even number of each $X \in\left\{X_{m+1}, \ldots, X_{n+n}\right\}$, so we will call an element $B \in \operatorname{Br}(\mathbf{X})$ bad if $\delta^{b}$ is even for each $b=m+1, \ldots, m+n$ and $\delta^{0}+\sum_{a=1}^{m} \delta^{a}$ is odd. $\mathrm{A}$ bracket is good if it is not bad. Note that these definitions of good and bad are slightly different than those that appeared in the paragraph before Proposition 2.1 . This difference will be addressed later in the proof. Let, $S_{m}$ denote the permutation group on $m$ symbols. For $\pi_{m} \in S_{m}$ and $\pi_{n} \in S_{n}$ define $\pi(B)$ to be the bracket obtained by fixing $X_{0}$, sending $X_{a}$ to $X_{\pi_{m}(a)}$ for $i=1, \ldots m$ and sending $X_{b}$ to $X_{\left(\pi_{n}(b-m)\right)+m}$ for $b=m+1, \ldots, m+n$. Now define the symmetrization operator

$$
\beta(B)=\sum_{\pi_{n} \in S_{n}} \sum_{\pi_{m} \in S_{m}} \pi(B) .
$$
by

Now, let $\theta \geq 1$ be a real number, and define $\Delta(\rho)$

$$
\begin{aligned}
& \Delta(\rho):\left(X_{0}, \ldots, X_{m+n}\right) \\
& \quad\left(\rho X_{0}, \rho^{\theta} X_{1}, \ldots, \rho^{\theta} X_{m}, \rho X_{m+1}, \ldots, \rho X_{m+n}\right)
\end{aligned}
$$

This dilation is compatible with $\hat{S}(X, K)$ by construction. Note also that this dilation makes each $\bar{\pi}$ a graded linear map. The $\Delta$-degree is given by

$$
\delta_{\theta}(B)=\delta^{0}(B)+\theta \sum_{i=1}^{m} \delta^{h_{i}}(B)+\sum_{i=1}^{n} \delta^{i+m}(B) .
$$

The following is a slightly simplified formulation of Theorem 4.1.

Proposition 5.1 Consider the bijection $\phi: \mathbf{X} \rightarrow \mathbf{f}$ which sends $X_{0}$ to $f . X_{a}$ to $h_{a}$ for $a=1, \ldots, m$, and $X_{b}$ to $g_{b-m}$ for $b=m+1, \ldots m+n$. Suppose that the system described by Equation 1 is such that every bad bracket $B \in B r(\mathbf{X})$ has the property that

$$
E v_{x:}(\phi)(\beta(B))=\sum_{a=1}^{k} \xi^{a} E v_{x}(\phi)\left(C_{a}\right)
$$

where $\xi^{i} \in \mathbb{R}$ and $\delta_{\theta}\left(C_{a}\right)<\delta_{\theta}(B)$ for $a=1, \ldots k$. Also suppose that 1 satisfies the LARC at $x$. Then the system described by Equation 1 is STLC at $x$.

Proof: Provided that the dilation defined by Equation 8 is compatible with $\hat{S}(\mathbf{X}, K)$ and that the collection of all $\bar{\pi}$ 's comprise a group which is an imput symmetry, then this is simply a restatement of Theorem 4.1. As previously mentioned, the dilation defined by Equation 8 is compatible with $\hat{S}(\mathrm{X}, K)$ by construction.

We need to show that the group comprised of all the $\pi s$ is an input symmetry. Define $\bar{\pi}^{\#}$ by $\bar{\pi}^{\#}(\exp Z)=$ $\exp (\hat{\bar{\pi}}(Z)) Z \in \hat{L}(\mathbf{X})$, where $\hat{\bar{\pi}}: \hat{L}(\mathbf{X}) \rightarrow \hat{L}(\mathbf{X})$ is given by $\hat{\pi}(Z)=\sum_{i=1}^{\infty} \bar{\pi}\left(P_{i}\right)$, if $Z=\sum_{i=1}^{\infty} P_{i}$, where each $P_{i}$ is homogeneons of degree $i$. Clearly. $\bar{\pi}^{\#}$ simply fixes $X_{0}$, sends $X_{a}$ to $X_{\pi_{m}(a)}$ for $\dot{s}=1 \ldots m$ and sends $X_{b}$ 
to $X_{\pi_{n}(b-m)+m}$ for $b=m+1, \ldots, m+n$ for each term in the infinite series.

Now, from Equation 6, we can write,

$$
\bar{\pi}^{\#}(S(t))=\sum_{I}\left(\int_{0}^{t} u_{I}\right) X_{\pi(I)}
$$

where, for $I=\left\{i_{1}, \ldots, i_{k}\right\}, \pi(I)=$ $\left\{\pi_{m n}\left(i_{1}\right), \ldots, \pi_{m n}\left(i_{k}\right)\right\}$, where $m n$ is either $m$ or $n$, depending upon whether $i \in\{1, \ldots, m\}$ or $i \in\{m+1, \ldots, m+n\}$ respectively. However,

$$
\sum_{I}\left(\int_{0}^{t} u_{I}\right) X_{\pi(I)}=\sum_{I}\left(\int_{0}^{t} u_{\pi^{-1}(I)}\right) X_{I}
$$

since the summation is over all possible multi indices $I$. Since $\pi^{-1}$ maps $K$ to $K$, it follows that $\bar{\pi}^{\#}$ maps $\hat{S}(X, K)$ to $\hat{S}(X, K)$.

Now, the proof to Proposition 2.1 follows easily.

First, scale the coefficients $\lambda_{i}$ and $\alpha_{j}$ in Equation 2 so that

$$
\sum_{i}\left|\lambda_{i}\right|+\sum_{j}\left|\alpha_{j}\right|=1
$$

Now, observe that if the system

$$
\dot{x}=f(x)+\bar{h}_{i}(x) v^{i}+\tilde{g}_{j}(x) u^{j}
$$

where $\bar{h}_{i}=\lambda_{i} h_{i}$ and $\bar{g}_{j}=\alpha_{j} g_{j}$, where $\lambda_{i}$ and $\alpha_{j}$ are from Equation 10, is controllable, then so is the system described by Equation 1 (because we have effectively further restricted the set of allowable control inputs).

Note that the definition of "good" and "bad" defined in this Appendix is slightly different from that defined in the paragraph before Proposition 2.1. In particular, Proposition 2.1 required $\sum_{i=1}^{m} \delta^{h_{i}}(B) \neq 1$, for $B$ to be bad, but this is not the case for a bad bracket as defined in the Appendix. Otherwise, the definitions of good and bad brackets correspond.

Thus, if $B$ is a bad bracket such that $\sum_{i=1}^{m} \delta^{h_{i}}(B) \neq$ 1, then Proposition 2.1 is simply a restatement of Proposition 5.1 where, instead of requiring the symmetrization of a bad bracket, defined in Equation 7 , to be $\Delta$ nentralized, each term in the sum which defines the symmetrization must be individually $\Delta$ neutralized.

Now if $B$ is such that $\sum_{i=1}^{m} \delta^{h_{i}}(B)=1$, then $\beta(B)$ is of the form

$$
\beta(B)=\sum_{\pi_{n} \in S_{n}} \bar{\pi}(\tilde{B})
$$

where where the single $X \in\left\{X_{1}, \ldots, X_{m}\right\}$ in $B$ is replaced by $X_{1}+\cdots+X_{m}$ in $\tilde{B}$ by the operation of $\sum_{\pi_{m} \in S_{m}} \bar{\pi}(B)$. Recall that, by assimption, $\sum_{i=1}^{m} \tilde{h}_{i}=$ $-\sum_{j=1}^{n} \grave{g}_{j}$. Thus, if we let $\hat{B}$ be the bracket $\tilde{B}$ with the term $X_{1}+\cdots+X_{m}$ replaced by $-\left(X_{m+1}+\cdots+X_{m+n}\right)$, then, we have $\operatorname{Ev}_{x}(\phi)(\beta(B))=\operatorname{Ev}_{x}(\phi)\left(\sum_{\pi_{n} \in S_{n}} \hat{B}\right)$. Since $\delta_{\theta}\left(\sum_{\pi_{n} \in S_{n}} \hat{B}\right)<\delta_{\theta}(\beta(B))$, the hypotheses of Proposition 5.1 is satisfied.

\section{Conclusions}

In this paper, we have presented and illustrated the application of a controllability test for control systems which may have inputs constrained to be non negative. Although technically difficult to prove, this result is relatively straightforward to use in applications. We note that the results contained herein can be improved. Roughly, we have treated the vector fields corresponding to the constrained inputs in a manner similar to that for the drift term. Because of the hypothesis expressed in Equation 2, it seems that it should be possible to treat the constrained inputs in a manner more analogous to that of the normal input terms.

Of course, there are many other important related problems, not the least of which is the control synthesis problem. The controllability test presented is useful mainly as a tool for analysis. Clearly solutions to problems such as stabilization and trajectory generation for this class of systems are extremely important and should be natural extensions of this work. Also, as previously mentioned, possibly averaging techniques could be used to obtain constructive controllability results.

\section{Acknowledgments}

The authors would like to thank Andrew Lewis for helpful discussions regarding the technical aspects of the proof presented in Appendix 4. This work was partially supported by a grant from the Office of Naval Research.

\section{References}

[1] Hector J. Sussmann. A sufficient condition for local controllability. Siam J. Control and Optimization, $16(5): 790 \cdots 802,1978$

[2] Hector J. Sussmann. A general theorem on local controllability. Siam J. Control and Optimization, 25(1): $158 \quad 194,1987$.

[3] K. M. Lynch and M. T. Mason. Stable pushing: Mechanics, controllability, and planning. International Journal of Robotics Research, to appear.

[4] N. E. Leonard. Averaging and Motion Control of Systems on Lie Groups. PhD thesis, University of Maryland, 1994. Institute for System Research.

[5] Hector J. Sussmanu. Lie brackets and local controllability: A sufficient condition for scalar input systems. Siam J. Control and Optimization, 21(5):686 713, 1983.

[6] Andrew D. Lewis. Aspects of Geometric Mechanics and Control of Mechanical Systems. Cds technical memorandum cit-cds 95-017, California Institute of Technology, April 1995.

[7] Thomas W. Hungerford. Algebra. Springer Verlag, 1974.

[8] Jean-Pierre Serre. Lie Algebras and Lie Groups. Springer Verlag, 1992. 